\title{
Networking Protocols for Wireless Sensor Networks with Mobile Sinks
}

\author{
Catalina Aranzazu Suescun and Mihaela Cardei, PhD. \\ Department of Computer \& Electrical Engineering and Computer Science \\ Florida Atlantic University \\ Boca Raton, Florida \\ Email: \{caranzazusue2014, mcardei\}@fau.edu
}

\begin{abstract}
Wireless sensor networks (WSN) have been widely used in many applications, such as climate monitoring systems, fire detection, ocean activity monitoring, and smart cities. These networks can be homogeneous or heterogeneous, depending on the types of wireless devices used. WSNs play an important role monitoring and reporting environment information and collecting surrounding context to send it to a sink. The sink acts as a bridge between the WSN and the final user. The sink could be static, such as a base station or a gateway node or could be mobile such as a drone or an user carrying a smartphone.

When the sink is mobile, the path to reach the sink from any node in the network becomes obsolete frequently. Then, the problem to address in this case is how to reach the sink, without drastically depleting the network energy resources.

This paper focuses on networking protocols used to report events to a mobile sink and presents the state of the art in mechanisms used to report data and events to a mobile sink.
\end{abstract}

Index Terms-Energy efficiency, Event reporting, Mobile sink, Networking protocols, Wireless sensor network.

\section{INTRODUCTION}

In the last 10 years a lot of research has been done in the use of wireless sensor networks to detect and collect information on the climate, animal activity, natural disaster and to monitor patients and smart cities.

Two important problems in wireless sensor networks are saving energy and efficient data collection. Sensor nodes are battery powered and it is often impossible to recharge them, therefore it is critical to save energy. Otherwise sensors die, resulting in network partitioning. Data collection is an important operation needed to report sensor measurements (such as a fire detection) to the sink.

When the sink is mobile, there is an additional issue in the network: having an active path to reach the sink. The most common mechanism to have a route to the sink is flooding the network with Route Request messages (RREQ). However, flooding the network is always expensive in terms of energy consumption. Researchers have been developing different mechanisms to report information to a mobile sink trying to avoid flooding the network.

The rest of the paper is organized as follows: Section II presents a background on wireless sensor networks. Section III shows the state of the art in the networking protocols for

Digital Object Identifier (DOI): http://dx.doi.org/10.18687/LACCEI2018.1.1.548 ISBN: 978-0-9993443-1-6

ISSN: $2414-6390$
WSNs with mobile sinks. Section IV presents a comparison of the works described in section III. Finally, Section V states the conclusions of the paper.

\section{WIRELESS SENSOR NETWORKS}

Wireless sensor networks are a versatile and non expensive option for monitoring applications. They play an important role in monitoring and reporting environmental information, and collecting surrounding context. A wireless sensor network contains multiple nodes with sensing components that communicate wirelessly.

A sensor is a device that can detect and measure changes in the temperature, pressure, light, smoke, movement or position. Usually multiple sensors are needed to detect events. This network could be even more accurate in the detection of events being heterogeneous with different types of sensors. Sensors collaborate to detect complex events, such as a forest fire, and transmit the aggregated information to a sink, which can be static, for example a base station, or could be mobile, similar to a drone or a forest ranger equipped with a smartphone, see Figure 1.

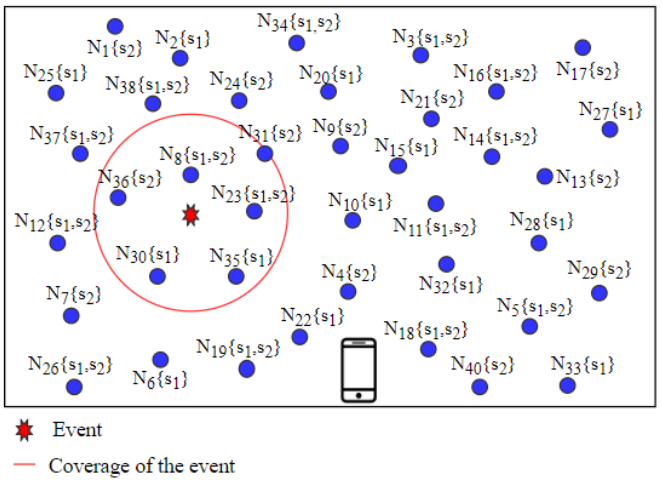

Fig. 1. Network deployment

In WSNs, an event is defined as an observable occurrence of a phenomenon or an object during a period of time in a specific area [1]. We distinguish two types of events [1]: atomic and composite events. Atomic events are defined as the measurement of changes in a single attribute in the 
environment, for example the smoke, while composite events are defined as a collection of atomic events.

These are several reasons why the nodes in a heterogeneous network have different sensing components [2]:

- nodes may be manufactured with different sensing capabilities

- some nodes may have purposely turned off some sensing components due to energy constraints

- some sensing components may fail over time

- some of the sensing components cannot be used due to lack of memory for storing data.

WSNs have limited capabilities in memory, battery and processing. When WSNs are deployed in hostile environments where human access is limited, and recharging or replacing wireless nodes is impractical or even prohibited in such situations, mechanisms for event detection and reporting have to minimize power consumption in order to prolong network lifetime.

Various mechanisms that save energy have been proposed in literature such as clustering, putting nodes to sleep during the periods of inactivity, and using reactive protocols.

\section{Networking Protocols}

The sink or sinks can have different mobility patterns: random (e.g. random walk), fixed or controlled. The following subsections present recent works in the development of routing protocols for WSN with mobile sink, when the path is random, fixed or controlled. Also we present some reactive protocols that help to prolong the life of the network by reducing the energy consumption.

\section{A. Mobile Sink with Random Walk}

The Anchor-based Voronoi-scoping Routing Protocol [3] considers several sinks that move using a random path approach. Each sink chooses an anchor from its neighbors, based on the nodes' signal strength. The anchor sends a hello message to the sink's Voronoi scope neighbors, so that they know how to send the information to the sink. The sink sends beacons to the anchor to maintain the link. If the signal strength between the sink and the anchor is low, then the sink chooses another node to be the anchor and the process repeats. The sink has a constant speed between 1 and $10 \mathrm{~m} / \mathrm{s}$. In the TRAIL protocol [3], the sink generates a trail of its movement through the network. A node that has messages for the sink uses a recent trail if it has one, or uses a random walk protocol to send data to the sink or to a sensor node that has a recent trail of the sink.

Article [2] proposes two protocols that use the concept of anchor, called Anchor-based Protocol and NewTree Protocol, see Figure 2 and two infrastructure-based algorithms, called Grid Flooding and Grid Sink-based Routing, see Figure 3.

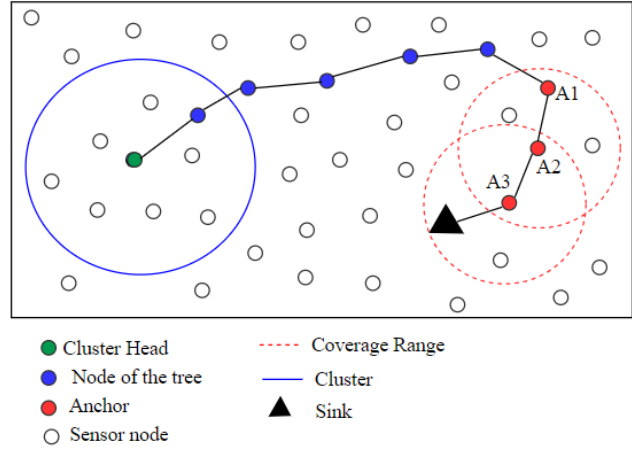

Fig. 2. Anchor selection

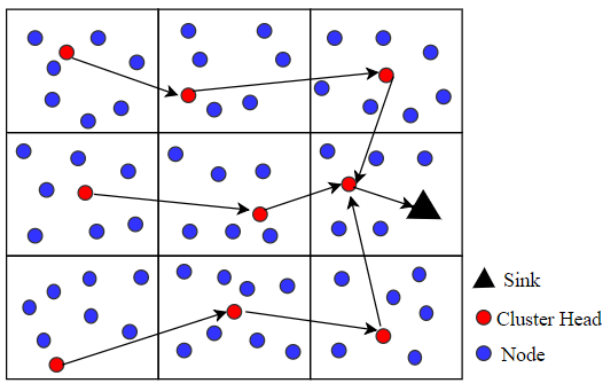

Fig. 3. Infrastucture-based algorithms

In Anchor-based Protocol, the sink $S$ selects the closest node as the first anchor, denoted $A_{1} . A_{1}$ then broadcasts a message in the whole network. As the message floods the network, a convergecast tree $T$ is formed, where $A_{1}$ is the root. Each node that receives the message for the first time, sets the sending node as its parent in $T$.

When one or more nodes start detecting the event, they initiate the mechanism for reporting the event. One or more clusters are formed using the event-based clustering algorithm from [2]. Event reports will flow from the nodes to the $\mathrm{CH}$, from $\mathrm{CH}$ to $A_{1}$ along $T$, and from $A_{1}$ to $S$. As long as $S$ is within communication range of $A_{1}$, no change is needed. To determine this, $A_{1}$ sends beacons (or data) periodically. If $S$ does not hear a beacon (or data) from $A_{1}$ for $\alpha$ periods (e.g. $\alpha=2$ ), then $S$ broadcasts a message to request a new anchor. Nodes which receive both $A_{1}$ 's beacons (or data) and $S$ 's message are candidates to become the second anchor $A_{2}$. When the first message is received by the sink, $S$ replies with an acknowledgement message, and that node becomes the second anchor. If $S$ moves out of the range of $A_{2}$, then the process repeats and a new anchor $A_{3}$ is selected. After the maximum number of anchors $\beta$ is reached, the anchor selection process resets, that means a new anchor $A_{1}$ is selected.

The NewTree-based Protocol mechanism is the same as Anchor-based Routing for $\beta=1$ anchor, and it follows the same framework. 
In the infrastructure-based algorithms the cluster head $(\mathrm{CH})$ is the node with the highest residual energy, and the cell size is set-up such that any $\mathrm{CH}$ is 1-hop neighbor with the $\mathrm{CH}$ of the nearby cells on horizontal and vertical directions. In this way, they do not need gateway nodes to ensure $\mathrm{CH}$ connectivity. In the Grid Flooding, data are flooded along the $\mathrm{CH}$ backbone, and as long as the sink is connected to at least one $\mathrm{CH}$, it will receive the message. In this case the sink moves inside the deployment area using a random walk, thus it will always be within communication range of at lest one $\mathrm{CH}$.

In Grid Sink-based Routing, the sink $S$ selects the closest $\mathrm{CH}$ as the root $R$. The root $R$ then broadcasts a message along the $\mathrm{CH}$ backbone. As the message is flooded along the backbone of CHs, a convergecast tree $T$ is formed, where $R$ is the root. The tree $T$ contains only $\mathrm{CH}$ nodes.

Article [4] also uses the concept of anchor or agent, which is a node closer to the sink. If the sink moves, then it waits a specific time $T$ to receive information from the anchor or agent node. If the sink does not receive any information, then it selects another agent node. The previous agent stores information for some time, until the sink broadcasts a message from the new location and new paths are formed, so the information can reach the sink. Source node paths are computed by the new agent using the Endocrine Cooperative Particle Swarm Optimization Algorithm. The algorithm computes a fitness function of the path that depends on three parameters: the energy of the nodes, the distance between nodes, and the communication delay. The path that has the largest fitness function has a more optimal path from the source node to the sink.

Another random path approach is Data Driven Routing Protocol [5]. There are three types of nodes, based on a given parameter $k$. O-nodes are 1-hop away from the sink, $M$ nodes are the nodes between 2 and $k$-hops away from the sink, and $I$-nodes are the nodes at distance at least $(k+1)$-hops away from the sink. When the sink moves, it sends beacon messages to its 1-hop neighbors. Beacon messages are resent in the network, so that the $O$-nodes and the $M$-nodes can update their routes to the sink. I-nodes send data to the sink using a random walk protocol until the first $M$-node or $O$ node is reached. Each route has an expiration time. $M$-nodes update their routes only if the time-stamp of the route is newer than the one stored in the memory. The nodes keep the older route in the memory as a backup, so two paths are stored in the routing table. This approach is used for one or multiple sinks.

\section{B. Mobile Sink with Predefined Path}

The Virtual Grid-based Dynamic Routes Adjustment scheme [6] is an infrastructure-based approach which partitions the network into fixed cells, where the node closest to the center is the cell-header. Adjacent cell-headers communicate via gateway nodes, which are normally located on the border of the clusters. Only cell-headers send information to the sink. When the sink moves through the network, the cell-headers adapt their path to the sink using the following mechanism. The sink sends beacons to the 1-hop cell-header which becomes the Originating Cell-Header $(\mathrm{OCH})$, see Figure 4. The $\mathrm{OCH}$ sets the sink as its next-hop. The $\mathrm{OCH}$ sends a route update to its neighbors called downstream cell-headers. In this way every cell-header has a route to reach the sink. The sink moves outside the field in counter-clockwise direction, with constant speed of $10 \mathrm{~m} / \mathrm{s}$.

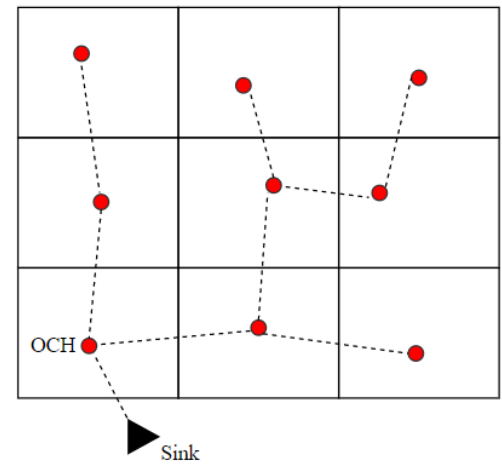

Fig. 4. Virtual Grid-based Dynamic Routes Adjustment scheme [6]

In the Mobile Sink based Adaptive Immune Energy Efficient Clustering Protocol [7], the network is divided into $R$ regions, where each region has the same number of nodes. The sink passes through each region and uses the adaptive immune algorithm to find its sojourn location and the location of the optimum cluster head. The communication range of each node is larger than the area of the region, so each node can send the information directly to the $\mathrm{CH}$. The $\mathrm{CH}$ s use Time Division Multiple Access to receive information from its cluster members to avoid possible collisions. The network also uses Code Division Multiple Access to avoid inter-cell interference. The nodes in the network sleep until the sink reaches its sojourn location in that region to reduce the energy consumption in the network. The path of the sink through the network is fixed and could be circular, rectangular, or linear, depending on how is the distribution of the regions.

In paper [8] the sink has a fixed movement path. The approach uses the concept of rendezvous points (RPs) see Figure 5, which are a subset of nodes that collect information from their neighbors. The RPs are similar to source nodes. The goal of the approach is to find a tour, using a Traveling Salesman Problem approach, which passes through all the RPs. This tour is used by the sink to collect data in the network. RPs are nodes spread in the networks to not be close from each other. In this way the information collected by the sink is not redundant. An optimal tour uses less RPs and covers the network with a minimal length.

Similarly to the previous work, the sink movement in [9] follows a predefined tour. The sink visits all the nodes in the area and collects data using 1-hop transmissions. The approach sets a specific number of polling points, where the sink moves to collect data. Using these polling points, the sink must cover all sensor nodes. The authors compute the movement tour 
of the sink through the network using a special case of the Traveling Salesman Problem. This heuristic approach involves building a minimum spanning tree and it runs in polynomial time.

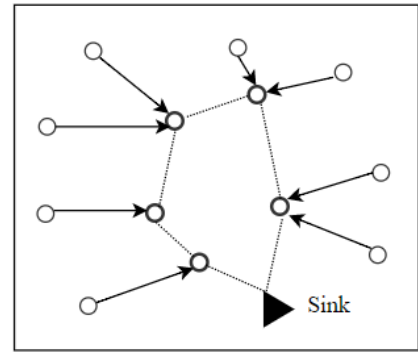

......... Sink Traveling path

O Rendezvous node

Fig. 5. Approach with Rendezvous Points [8]

In [10] the sink moves along a circular path, centered in the middle of the area. The sink moves only when the nearby sensor nodes have less energy than some predefined threshold. This results in less sink movements and less route update messages flowing through the network. The nodes hat do not need to send information to the sink, will sleep until the sink reach the region. The nodes use Carrier Sense Multiple Access/Collision Avoidance protocol (CSMA/CA) to avoid collision in the network.

Article [11] presents also a protocol with circular path, called Ring Routing Protocol that has three types of nodes: ring, anchor, and regular nodes. Ring nodes are within a specific distance from the center of the network and they store information about the position of the anchor, see Figure 6.

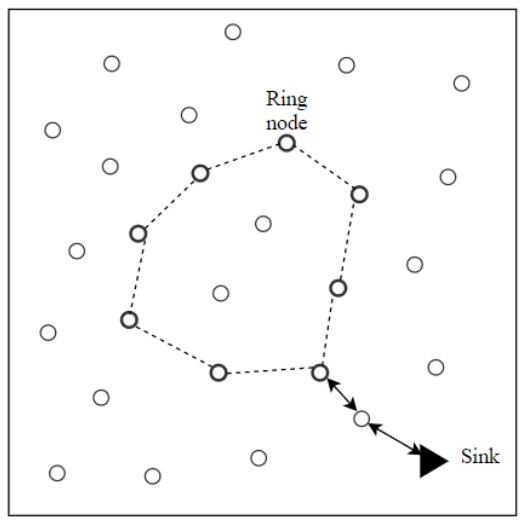

Fig. 6. Ring Routing protocol [11]

The anchor is the closest node to the sink and is renewed each time the link quality with the sink is lower than some threshold. The information of the new anchor is then sent to the ring nodes. If an old anchor receives messages addressed to the sink, then it forwards the packets to the new anchor.
When a regular node wants to send information to the sink, it requests the position of the anchor from the ring nodes. The node then sends the information to the anchor using geographical routing. There is no limit on the number of anchors used in the network.

\section{Mobile Sink with Controlled Path}

In [12], the sink location is based on the energy of the network. Sink movement is controlled by a genetic algorithm that calculates a population of chromosomes. Each chromosomes represents a sensor and has two parts: $\mathrm{X}$ that is the length of the network environment, and $\mathrm{Y}$ is the width of network environment. Each chromosome is evaluated by a fitness equation that depends on the energy of each sensor and the distance between the sink and the sensor. A chromosome is selected if it has the highest probability, computed as the ratio of the fitness of the chromosome and the sum of the fitness of all chromosomes. In this way, the sink will move to the position of the chromosome selected that has the maximum residual energy. The genetic algorithm is an extension of the LEACH [13] algorithm, resulting in an energy aware version.

Paper [14] proposes algorithms for data gathering when the sinks move along fixed paths and controlled paths. In the fixed path approach, the area is divided in hexagonal crowns, where the sinks move along the hexagonal perimeters, and they stop periodically to collect data in each corner of the hexagon. In the controlled path approach, every time the sink reaches a new position, form a cluster with the sink as Cluster Head. When the energy level of the sink 1-hop neighbors drops under a threshold, the sink broadcast a message to find a new position to collect information. The sinks are interconnected all the time, forming a virtual backbone.

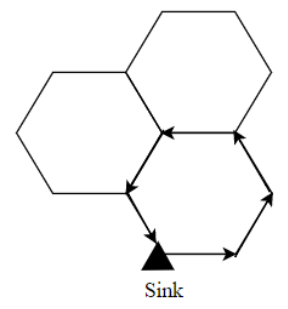

Fig. 7. Hexagonal crowns approach [14]

Another controlled sink mechanism using a biased random walk path is presented in [15]. The sinks and the source nodes know their locations. If an event occurs, the sink selects a rendezvous node and sends a query packet (Qpacket). Qpacket has a counter that starts at zero and is incremented by 1 every time a node rebroadcasts it. If a node receives a Qpacket with the counter equal to or greater than one, then it checks with its neighbors that have also received the Qpacket with counter greater than one, in order to select who is going to rebroadcast the packet. The node selected has the smallest Qpacket arrival time, that means a shortest path. When the source receives the Qpacket, it sends a replay packet (Rpacket) to all the neighbors 
and they rebroadcast the message to the rendezvous node. If the sink is close to the rendezvous node, then the rendezvous node receives the Rpacket directly from the sink. If the sink is out of the communication range of the rendezvous node, then it selects a relay node to reach the rendezvous node. If an event occurs, then the nodes sensing this event collectively process the signal and select one node as the source. The rendezvous node transmits packets to the sink every period. If the source does not have any packet to transmit, then it sends a null packet to the sink. If the sink does not receive any packet for a specific time from the rendezvous node, then it selects a relay node to communicate with the rendezvous node. Then, if the sink is out of the range of the relay node, it selects a new relay node and sends a message to the old relay node in order to eliminate it.

\section{Reactive Protocols for WSNs with Mobile Sinks}

An important observation is that the nodes in the network may not always have data or events to report. Then, mechanisms such as clustering, sleep mode for inactive sensors, and the use of active/reactive protocols based on the application type, can be used to save energy.

Proactive protocols are more suitable for periodic or continuous data reporting, while reactive protocols are more appropiate for event-based data reporting and mobile events. In proactive protocols, nodes maintain updated routing information all the times. This usually leads to high overhead triggered by frequent control messages. In contrast, reactive protocols update routing information only when needed (e.g. when reporting data). Such an approach usually reduces the overhead at the cost of an increased delay for establishing a path to the destination [16].

Several research works have proposed reactive protocols. Most of the authors use Route Request Query (RREQ) and Route Reply (RREP) messages to discover a route to the sink. HELLO messages are used to maintain updated information about neighbors and about the paths to the sink. These protocols are inspired by Ad Hoc On-demand Distance Vector Routing (AODV) [17] and Dynamic Source Routing (DSR) [18] routing protocols developed for Ad Hoc wireless networks. Starting from these protocols, several developments can be found in the academic field [19]-[21].

In [19], the concept of "gateway" is used. The area has a stripe, cross section in the middle, called the "rendezvous region", see Figure 8 . The nodes in this region form a backbone used to transfer information to the sink. Every node has a neighbor table with its one hop neighbors' information. If a node is in the boundary of the rendezvous area, then it can start the tree formation. In order to send a message, the boundary node selects from its neighbor table the neighbor that has the largest residual energy and is located closer to the centroid of the network.

Every node that receives the message, repeats the process until the node that is the centroid of the rendezvous area is reached. If a node that is out of the cross section has to send information to the sink, then it sends the message to the closest backbone node. When the sink wants to collect information from the network, it reaches a new point and selects a gateway node. The gateway sends an ACK to its neighbors and each neighbor re-sends the ACK. The process is repeated until the message reaches a backbone node that replies to the ACK such that the path can be created. Also, when the sink moves, it can send its location to the network using backbone nodes. If any node has to send information to the sink, it can send a request to the backbone nodes and they will reply with the location of the sink.

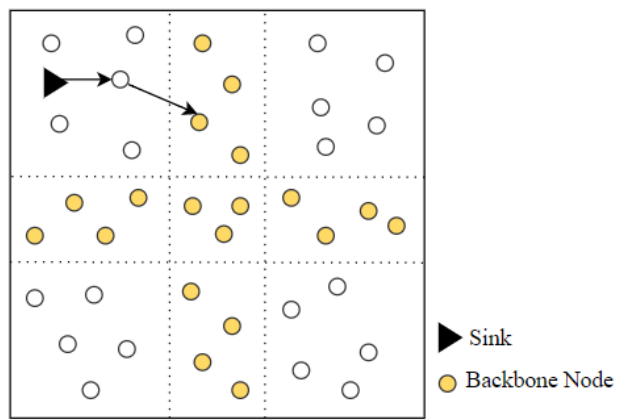

Fig. 8. Rendezvous Region (Cross section) approach [19]

In article [20] the authors develop an anycast tree based routing protocol with multiple mobile sinks. The nodes are mobile. The authors use Rayleigh's law to compute the distance between nodes. The communication range of a node in every quadrant depends on the environment. If a node needs to send information to the sink, then it sends a RREQ with a Time To Live (TTL) set to 1 . If the sink is 1-hop away, then the sink replies to the node. If the node does not receive the RREP after some specific time, then it sends a new RREQ and increments the TTL by 1 . If the node does not receive a RREP after the maximum increase of the TTL, then the communication is considered unsuccessful. Based on the RREP messages, a convergecast tree is formed, which is used to send information to the sink. Messages from nodes to the sink and the RREP packets are transmitted in unicast mode. If a node sends a RREQ and receives a RREP from a sink, then it stores that route and discards the other RREP messages from other sinks. If a node receives a RREQ, then it checks if it has a path to a sink and if it has one, then it uses the unicast mode to resend the information to the next node in the path. If the node does not have a path, then it broadcasts the message using the increasing TTL process. Using the anycast model, reduces the message transmission in the network and also the energy consumption.

The authors of [21], develop three modules: route discovery, forwarding decision, and forwarder selection and prioritization. If a node needs to send a message to the sink and it does not have a recent route, then the node starts a route discovery using a Route Request (RREQ) message. When nodes receive RREQ for first time, they store the sender ID and 
the sequence number. Each node computes a time delay before rebroadcasting the RREQ. The delay is computed by dividing the number of hops to the source to the sum of the packet reception ratio of the common neighbors. In this way, a node with better link quality with its neighbors, more forwarding candidates, and shorter path to the source has a lower delay timer.

When the destination receives the RREQ, it replies with Route Reply (RREP) message along the route of the first message. When the RREP is sent, every node on the path attaches its possible candidates (for forwarding the message) and their priorities, such that the source node has more options if some intermediate node of the current route fails. The source node sends the message to the sink by broadcasting the message to all the possible forwarder nodes. Each node that receives the message initiates the delay timer. When the timer expires and the node did not receive an Acknowledgement (ACK) message, it sends an ACK to the message, so that other potential senders do not rebroadcast the message. To evaluate the performance of their protocol, the authors implement the protocol as an extension of AODV [17]. They compare their protocol with Geographic Opportunistic Routing Protocol (GOR), AODV-EXT and Reliable and Efficient Packet Forwarding (REPF) algorithm, obtaining similar performance to the GOR protocol.

In paper [22], an energy-efficient hybrid routing protocol is presented. The sink broadcasts its location every $T$ seconds to its "proactive area" defined over $N$ hops. $N$ depends on the network size and data dissipation. The nodes that receive the message from the sink and are within the first $N / 2$ hops from the sink always rebroadcast the sink message. If the nodes are in the final $N / 2$ hops from the sink, they only rebroadcast the message when one of two possibilities occur: an internal counter is greater than some predefined threshold or a random number $M$ calculted is greater than some threshold. The nodes that did not receive the message from the sink are in the "reactive area" and if they need to send information to the sink, they will broadcast a RREQ message to their $h-h o p s$ neighbors. Broadcasting of the route request message depends also on $M$ and the distance to the sink. The distance to the sink is computed using the history of movements of the sink and its current position. When the route request message reaches a node in the "proactive area", then the node sends a route RREP using the reverse path. RREP is also used by the neighbors to extend the knowledge of the path in the network and to reduce possible new RREQ. If more reverse paths are generated, then the one with less hops is used.

Authors of [23], present a reactive version of their anchorbased protocol of [2]. In this case a time-out procedure is implemented. If no data (event reports) are sent for some periods of time, then the fields for the parents in the tree are considered obsolete and removed. Depending on the length of the period without event, the fields may be deleted or not. If the parent fields are not obsolete, then data flows from $\mathrm{CH}$ to $A_{1}$ along $T$, and from there to $S$ along the chain of anchors. Also to save energy and improve event reporting delay, they implement a shortcut mechanism. If a node in $T$ receives beacons from an anchor, then the node stores this anchor as its parent. Then the node is sending data directly to the anchor instead of sending it through the rest of the path. This mechanism is illustrated in Figure 9.

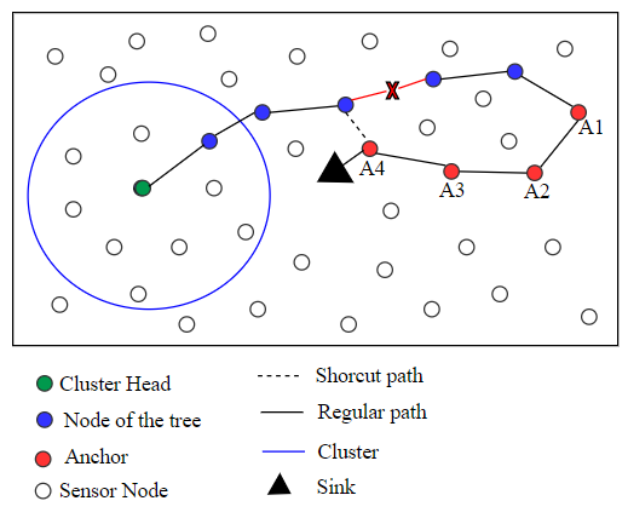

Fig. 9. Shortcut mechanism

\section{ANALYSIS OF THE STATE OF THE ART}

Table I presents a summary of characteristics of the works described in Section III.

Table II presents some of the drawbacks detected in the related works of Section III and also a possible solution to each issue.

\section{Conclusions}

This paper presents the state of the art in networking protocols for wireless sensor networks with mobile sinks.

Some conclusions for this paper are:

- When the sink is mobile, the path to reach the sink from any node in the network becomes obsolete very frequently. Therefore it is important to design energyefficient mechanisms which reconstruct the paths to the sink when is needed.

- Several works have been proposed related to in this topic, using innovative mechanisms such as anchor nodes, relay nodes, and rendezvous points, to reduce the consumption of energy in the network.

- The use of clustering mechanisms can help in the reduction of energy consumption in the networks. Even-based clustering can further reduce the energy consumption since a smaller number of clusters are expected to be formed and to report data to the sink.

- Additional research is needed to combine energy-efficient mechanisms with techniques which improve accuracy, response time and the overall performance of the network. 
TABLE I

COMPARISON TABLE OF RELATED WORKS

\begin{tabular}{|c|c|c|c|c|c|c|}
\hline $\begin{array}{l}\text { Work / } \\
\text { Characteris- } \\
\text { tic }\end{array}$ & $\begin{array}{lr}\text { Anchor } & \text { nodes, } \\
\text { Beacons } & \text { and } \\
\text { ACK } & \\
\end{array}$ & Clustering & $\begin{array}{l}\text { Single or multi- } \\
\text { ple sinks }\end{array}$ & $\begin{array}{l}\text { Known } \\
\text { algorithms } \\
\text { Used }\end{array}$ & $\begin{array}{l}\text { Sink mobility } \\
\text { pattern }\end{array}$ & $\begin{array}{l}\text { Proactive or re- } \\
\text { active }\end{array}$ \\
\hline Tian [3] & $\begin{array}{l}\text { Multiple anchors, } \\
\text { Beacons and } \\
\text { ACK }\end{array}$ & No clustering & Multiple sinks & Voronoi Scoping & Random walk & Proactive \\
\hline Aranzazu [2] & $\begin{array}{l}\text { Limited number } \\
\text { of anchors and } \\
\text { beacons from an- } \\
\text { chor }\end{array}$ & $\begin{array}{l}\text { Clustering } \\
\text { approach and } \\
\text { Infrastucture } \\
\text { based approach }\end{array}$ & Single sink & No & Random walk & Proactive \\
\hline Hu [4] & One anchor & No clustering & Single sink & $\begin{array}{l}\text { Endocrine } \\
\text { Cooperative } \\
\text { Particle Swarm } \\
\text { Optimization } \\
\text { Algorithm }\end{array}$ & Random walk & Proactive \\
\hline Shi [5] & $\begin{array}{l}\text { No anchors. The } \\
\text { sink sends bea- } \\
\text { cons. }\end{array}$ & No clustering & Multiple sinks & No & Random walk & Proactive \\
\hline Khan [6] & $\begin{array}{l}\text { No anchors. The } \\
\text { sink sends bea- } \\
\text { cons }\end{array}$ & $\begin{array}{l}\text { Yes, } \\
\text { infrastructure } \\
\text { based }\end{array}$ & Single sink & No & Fixed (circular) & Proactive \\
\hline Abo [7] & No anchors & $\begin{array}{l}\text { Yes, } \\
\text { infrastructure } \\
\text { based }\end{array}$ & Single sink & $\begin{array}{l}\text { Adaptive } \\
\text { Immune } \\
\text { algorithm }\end{array}$ & $\begin{array}{l}\text { Fixed (circular, } \\
\text { rectangular or } \\
\text { linear) }\end{array}$ & Proactive \\
\hline Salarian [8] & No anchors & Yes & Single sink & $\begin{array}{ll}\text { Heuristic } & \text { for } \\
\text { Traveling } & \\
\text { Salesman } & \\
\text { Problem } & \end{array}$ & Fixed (a tour) & Proactive \\
\hline Ma [9] & No anchors & No clustering & Single sink & $\begin{array}{l}\text { Heuristic for } \\
\text { Traveling } \\
\text { Salesman } \\
\text { Problem and } \\
\text { Minimum } \\
\text { Spanning Tree }\end{array}$ & Fixed (a tour) & Proactive \\
\hline Khan [10] & No anchors & No clustering & Single sink & $\begin{array}{l}\text { Carrier Sense } \\
\text { Multiple } \\
\text { Access/Collision } \\
\text { Avoidance } \\
\text { protocol }\end{array}$ & Fixed (circular) & Proactive \\
\hline Tunca [11] & $\begin{array}{l}\text { Unlimited num- } \\
\text { ber of anchors }\end{array}$ & No clustering & Single sink & No & Fixed (circular) & Proactive \\
\hline Patel [12] & No anchors & No clustering & Single sink & $\begin{array}{l}\text { Genetic } \\
\text { algorithm }\end{array}$ & Controlled & Proactive \\
\hline Marta [14] & No anchors & $\begin{array}{l}\text { Yes, the sink is } \\
\mathrm{CH}\end{array}$ & Multiple sinks & No & $\begin{array}{l}\text { Fixed and con- } \\
\text { trolled }\end{array}$ & Proactive \\
\hline Perumal [15] & $\begin{array}{l}\text { Anchors called } \\
\text { rendezvous and } \\
\text { relay nodes }\end{array}$ & Yes & Multiple sinks & No & $\begin{array}{l}\text { Controlled } \\
\text { (biased random } \\
\text { walk) }\end{array}$ & Proactive \\
\hline Sharma [19] & $\begin{array}{l}\text { Anchor called } \\
\text { gateway node }\end{array}$ & No clustering & Single sink & No & Random walk & Reactive \\
\hline Kostin [20] & No anchors & No clustering & Multiple sinks & $\begin{array}{l}\text { Rayleigh's law } \\
\text { for distance }\end{array}$ & Fixed & Reactive \\
\hline Niu [21] & No anchors & No clustering & Single sink & AODV & Random walk & Reactive \\
\hline Kaleibar [22] & No anchors & No clustering & Single sink & No & Fixed & $\begin{array}{l}\text { Hybrid (proactive } \\
\text { and reactive) }\end{array}$ \\
\hline $\begin{array}{l}\text { Aranzazu } \\
{[23]}\end{array}$ & $\begin{array}{l}\text { Limited number } \\
\text { of anchors and } \\
\text { beacons from an- } \\
\text { chor }\end{array}$ & Clustering & Single sink & No & Random walk & Reactive \\
\hline
\end{tabular}

16th LACCEI International Multi-Conference for Engineering, Education, and Technology: Innovation in Education and Inclusion, 18-20 July 2018, Lima, Peru. 
TABLE II

COMPARISON TABLE OF RELATED WORKS

\begin{tabular}{|c|c|}
\hline Issues & $\begin{array}{l}\text { Possible solutions } \\
\end{array}$ \\
\hline $\begin{array}{l}\text { the use of two-way messages to maintain the link between the sink } \\
\text { and the anchor may result in wasting the energy in the network }\end{array}$ & use one way beacons to maintain the link \\
\hline $\begin{array}{l}\text { the use of an unlimited number of anchors results in a long path to } \\
\text { the sink }\end{array}$ & $\begin{array}{l}\text { use a limited number of anchor nodes to deal with the sink mobility } \\
\text { and use mechanisms to shorten the path from the } \mathrm{CH} \text { to the sink }\end{array}$ \\
\hline $\begin{array}{l}\text { the use of complicated algorithms in the nodes may require a powerful } \\
\text { processor }\end{array}$ & $\begin{array}{l}\text { the processing of complicated algorithms could be done in a powerful } \\
\text { device outside the network }\end{array}$ \\
\hline $\begin{array}{l}\text { the use of multiple sinks results in multiple routes and more energy } \\
\text { consumption in the network }\end{array}$ & $\begin{array}{l}\text { join route mechanisms could be used to reduce the number of paths } \\
\text { in the network and to reduce the energy consumption }\end{array}$ \\
\hline $\begin{array}{l}\text { the use of proactive approaches can waste energy in the network if } \\
\text { the network may not always have data or events to report }\end{array}$ & $\begin{array}{l}\text { use reactive mechanisms to avoid sending messages when no event is } \\
\text { present }\end{array}$ \\
\hline some works present a sink that walks outside the monitoring area & the sink should be inside the area to have a more realistic scenario \\
\hline $\begin{array}{l}\text { most of the papers assume a constant speed for the sink, which is not } \\
\text { realistic }\end{array}$ & the speed of the sink needs to change to match with a realistic model \\
\hline $\begin{array}{l}\text { use of fixed clustering approach, generate more consumption of energy } \\
\text { if the event spans multiple grid cells and if the composite event } \\
\text { involves sensing components from nodes located in neighboring grid } \\
\text { cells, then only the sink has all the information needed to detect the } \\
\text { composite event }\end{array}$ & $\begin{array}{l}\text { Event-based clustering mechanisms can detect the composite event } \\
\text { earlier, as result of aggregation }\end{array}$ \\
\hline
\end{tabular}

\section{REFERENCES}

[1] J. Gao, J. Li, Z. Cai and H. Gao. Composite event coverage in Wireless Sensor Networks with Heterogeneous Sensors. INFOCOM 2015.

[2] C. Aranzazu Suescun, and M. Cardei. Distributed algorithms for event reporting in mobile-sink WSNs for Internet of Things. Tsinghua Science and Technology Journal. DOI: 10.23919/TST.2017.7986944. August, 2017.

[3] K. Tian, B. Zhang, K. Huang, and J. Ma. Data gathering protocols for wireless sensor networks with mobile sinks. Proceedings IEEE GLOBECOM10, 2010.

[4] Y-F. Hu, Y-S. Ding, L-H. Ren, K-R. Hao, and H. Han. An endocrine cooperative particle swarm optimization algorithm for routing recovery problem of wireless sensor networks with multiple mobile sinks. Information Sciences, Vol. 300, DOI: 10.1016/j.ins.2014.11.052, Apr. 2015.

[5] L. Shi, B. Zhang, H. T. Mouftah, and J. Ma. DDRP: an efficient data-driven routing protocol for wireless sensor networks with mobile sinks. International Journal of Communication Systems. DOI: $10.1002 /$ dac. 2315, 2013.

[6] A. W. Khan, A. H. Abdullah, M. A. Razzaque, and J. I. Bangash. VGDRA: a virtual grid-based dynamic routes adjustment scheme for mobile sink-based wireless sensor networks. IEEE Sensors Journal, Vol. 15, No. 1, Jan. 2015.

[7] M. Abo-Zahhad, S. M. Ahmed, N. Sabor, and S. Sasaki. Mobile sinkbased adaptive immune energy efcient clustering protocol for improving the lifetime and stability period of wireless sensor networks. IEEE Sensors Journal, Vol. 15, No. 8, Aug. 2015.

[8] H. Salarian, K-W. Chin, and F. Naghdy. An energy-efficient mobile-sink path selection strategy for wireless sensor networks. IEEE Transactions on Vehicular Technology, Vol. 63, No. 5, Jun. 2014.

[9] M. Ma, Y. Yang, and M. Zhao. Tour planning for mobile data-gathering mechanisms in wireless sensor networks. IEEE Transactions on Vehicular Technology, Vol. 62, No. 4, May 2013.

[10] M. I. Khan, W. N. Gansterer, and G. Haring. Static vs mobile sink: the influence of basic parameters on energy efficiency in wireless sensor networks. Computer Communications Journal, 2012.

[11] C. Tunca, S. Isik, M. Y. Donmez, and C. Ersoy. Ring routing: an energyefficient routing protocol for wireless sensor networks with a mobile sink IEEE Transactions on Mobile Computing, Vol. 14, No. 9, Sep. 2015.

[12] B. Patel and D. Bhagat. Shifting of sink position in wireless sensor network. International Journal of Engineering Development and Research, Vol. 2, Issue 2, 2014

[13] W. R. Heinzelman, A. Chandrakasan, and H. Balakrishnan. Energyefficient communication protocol for wireless microsensor networks. Proceedings of the 33rd Hawaii International Conference on System Sciences, Jan. 2000.
[14] M. Marta and M. Cardei. Improved sensor network lifetime with multiple mobile sinks. Elsevier Journal of Pervasive and Mobile Computing, Vol. 5, No. 5, pp. 542-555, Oct. 2009.

[15] M. Perumal and S. Dhandapani. Modeling and simulation of a novel relay node based secure routing protocol using multiple mobile sink for wireless sensor networks. The Scientific World Journal. DOI: $10.1155 / 2015 / 495945$. August, 2015.

[16] A. Koliousis and J. Sventek. Proactive vs reactive routing for wireless sensor networks. http://www.doc.ic.ac.uk/ akolious/documents/TR-20 07-293.pdf. 2007.

[17] C. Perkins and E. Belding-Royer and S. Das. Ad hoc on-demand distance vector (AODV) routing. Network Working Group Journal. https://tools.ietf.org/html/rfc3561. July, 2003.

[18] D. Johnson and Y. Hu and D. Maltz, The dynamic source routing protocol (DSR) for mobile ad hoc networks for IPv4. Network Working Group Journal. https://tools.ietf.org/html/rfc4728. February 2007.

[19] S. Sharma and D. Puthal and S. Kumar Jena and A. Y. Zomaya and R. Ranjan. Rendezvous based routing protocol for wireless sensor networks with mobile sink. The Journal of Supercomputing. DOI: 10.1007/s11227016-1801-0. March 2017.

[20] A. E. Kostin and Y. Fanaeian and H. 1-Wattar. Anycast tree-based routing in mobile wireless sensor networks with multiple sinks. Wireless Networks Journal. DOI : 10.1007/s11276-015-0975-3. February, 2016.

[21] J. Niu and L. Cheng and Y. Gu and L. Shu and S. K. Das. R3E: Reliable reactive routing enhancement for wireless sensor networks. IEEE Transactions on Industrial Informatics Journal. DOI:10.1109/TII.2013.2261082. February, 2014.

[22] F. J. Kaleibar and M. Abbaspour and H. S. Aghdasi. An energy-efficient hybrid routing method for wireless sensor networks with mobile sink. Wireless Personal Communication Journal. DOI: $10.1007 / \mathrm{s} 11277-016-3434-2$. October 2016.

[23] C. Aranzazu-Suescun and M. Cardei. Reactive routing protocols for event reporting in mobile-sink wireless sensor networks The 13th ACM Symposium on QoS and Security for Wireless and Mobile Networkss. November 2017. 